\title{
HI-DISTRIBUTION, KINEMATICS AND GEOMETRY OF THE LARGE MAGELLANIC CLOUD
}

\author{
K. ROHLFS, Th. LUKS \\ Astronomical Institute \\ Ruhr-University \\ 4630 Bochum 1 \\ F. R. Germany
}

ABSTRACT. Here we discuss current observational data on, and the distribution of, neutral hydrogen in the Large Magellanic Cloud (LMC).

\section{Observations}

The fact that the $64 \mathrm{~m}$ radiotelescope at Parkes is still the largest single dish in the southern hemisphere capable of measuring $\mathrm{HI} 21 \mathrm{~cm}$ line radiation is the reason why all "high" - resolution surveys have the same $H P B W=14.9^{\prime}$. This corresponds to a linear resolution of $215 \mathrm{pc}$ in the LMC if a distance to the galaxy of $49.4 \mathrm{kpc}$ is adopted. New surveys therefore have become expedient only because of significant advances in receiver technology.

General surveys so far undertaken include:

- McGee \& Milton (1966a, b) $B=33 \mathrm{kHz}, \Delta T \approx 1 K$;

- Rohlfs et al.(1984) B $=1 \mathrm{kHz}, \Delta T \leq 0.08 \mathrm{~K}$, survey field $6.2^{\circ} \times 6.2^{\circ}$, sampling grid $0.2^{\circ} \times 0.2^{\circ}$;

- Rohlfs \& Luks (1990) B $=1 \mathrm{kHz}, \Delta T \leq 0.05 K$, survey field $8.2^{\circ} \times 8.2^{\circ}$, sampling grid

$0.2^{\circ} \times 0.2^{\circ}$

The 1990 extension of the LMC survey of Rohlfs et al. (1984) has been performed with identical specifications regarding the receiver configuration, calibration and reduction procedure and arrangements of the sampling grid. Therefore, the two data sets should form one homogeneous sample.

Specific regions of the Cloud have been surveyed by Dopita et al. (1985) and Meaburn et al. (1987). $21 \mathrm{~cm}$ line profiles observed are generally dominated by a single peak, which is, however, quite often supplemented by one or several additional minor peaks with radial velocities that differ by up to $40 \mathrm{~km} \mathrm{~s}^{-1}$.

\section{The distribution of the column density}

There is strong line-emission over an area of roughly $7^{\circ} \times 8^{\circ}$, the larger extent being in the N-S direction, and the most striking fact about the brightness distribution is that it bears no resemblance

R. Haynes and D. Milne (eds.), The Magellanic Clouds, 63-67.

(C) 1991 IAU. Printed in the Netherlands. 
at all to the appearance of the LMC. In particular, the optical bar region is not visible at all in the HI distribution, and the most conspicuous feature of the neutral hydrogen distribution, the large cloud extending for about $4^{\circ}$ to the south from 30 Dor, has no counterpart in the stellar distribution. About $16 \%$ of the HI content of the LMC is contained in this cloud, and it thus dwarfs all other HI features.

Comparing the HI column density distribution (Figs. 1,3 and 4) with that of the CO molecule as given by the survey of Cohen et al. (1988), we find a fairly good correlation, although there is no tight functional relation. Usually, HI clouds have a counterpart in the $\mathrm{CO}$ distribution, but there are both HI peaks without $\mathrm{CO}$ maximum and vice versa in this case.

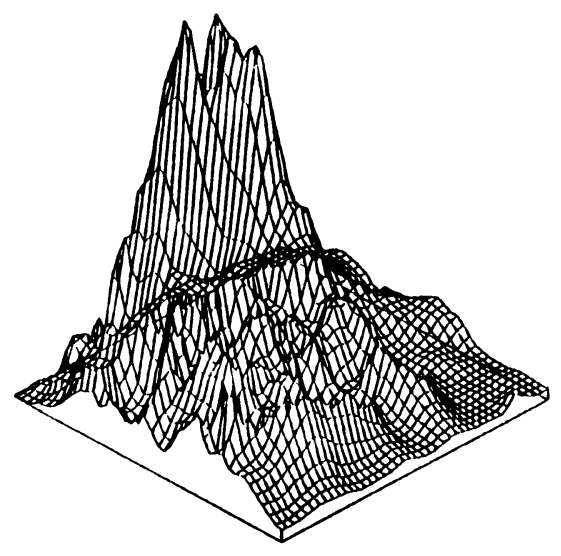

Figure1. A ruled-surface plot of the column density distribution of $\mathrm{HI}$ for the LMC.

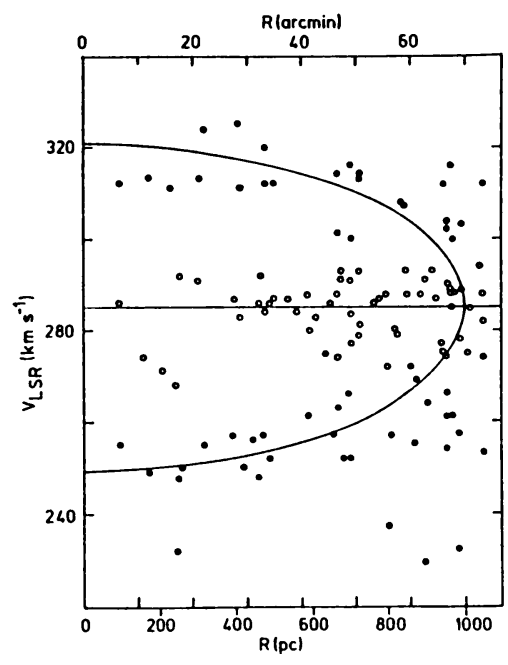

Figure 2. Radial velocity of the Gaussian components for the HI material in the constellation Shapley III. An expanding shell with $v_{\text {exp }}=36 \mathrm{~km} \mathrm{~s}^{-1}$ and diameter $d=2.0$ kpc has been fitted (after Dopita et al. 1985).

Turning to the distribution of emission nebulae and $\mathrm{H} \alpha$ regions (Davies et al. 1976, Feitzinger et al. 1977) or late type super giants (Martin et al. 1989) the correlation gets less and less tight although it definitely still exists. In particular, the large HI cloud south of 30 Dor contains few emission nebulae and no late type supergiants for angular distances $\geq 1.5^{\circ}-2^{\circ}$ from 30 Dor.

One feature seen clearly both in the distribution of HI, HII and early type stars is the void in the gas distribution at $\alpha_{1950}=5^{h} 31^{m}, \delta_{1950}=-66^{\circ} 54^{\prime}$ first identifiedwith the constellation Shapley III by Westerlund (1964). It was extensively investigated by Dopita et al. (1985), who explain it in terms of an expanding ring of $\mathrm{HI}$ gas with a radius of $\mathrm{d}=1.8 \mathrm{kpc}$ and expansion velocity $36 \mathrm{~km} \mathrm{~s}^{-1}$. This model thus explains the observed shape of the HI line profiles in this region that consist of a main central component framed by two smaller components with a brightness of about $10 \%$ of the central one and a radial velocity differing up to $35-40 \mathrm{~km} \mathrm{~s}^{-1}$. Concentric with this ring of $\mathrm{HI}$ gas is an $\mathrm{HII}$ ring with $\mathrm{d}=1.4 \mathrm{kpc}$ expansion velocity $30 \mathrm{~km} \mathrm{~s}^{-1}$. The supergiant loop LMC 4 follows this feature equally well. It can be best understood in terms of propagating star formation over the last $1.5 \times 10^{7}$ years with the diameter of the star forming region increasing from $300 \mathrm{pc}$ to $1200 \mathrm{pc}$ during this time interval. 


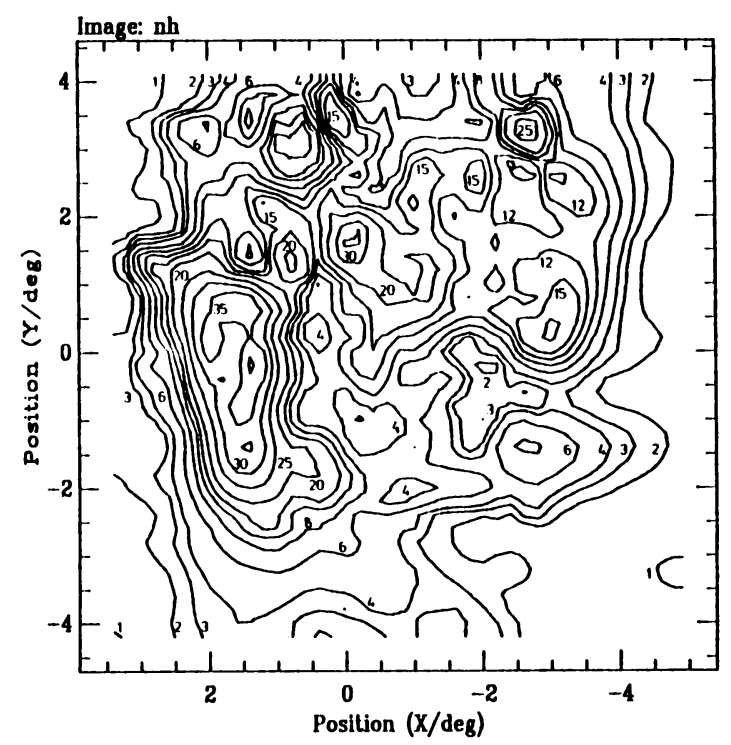

Figure 3. The distribution of neutral hydrogen column density with coordinates in degrees from a fixed position in the LMC. Contour levels at $1,2,3,4,6,8,10,12,15,20,30,35 \times 10^{20} \mathrm{~cm}^{-2}$

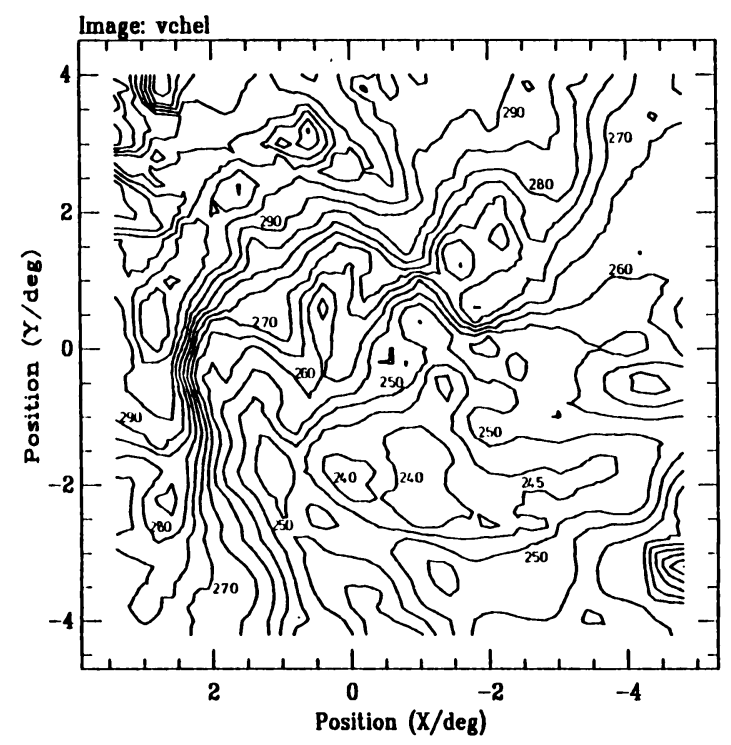

Figure 4. Iso-velocity contours of the intensity weighted mean heliocentric radial velocity. 
It is not clear whether another void in the HI distribution centred at $\alpha_{1950}=5^{h} 27^{m}, \delta_{1950}=$ $-68^{\circ} 52^{\prime}$ can be explained in a similar way. Neither the distribution of the HII gas nor the distribution of the young stars shows one as clearcut as that of LMC 4.

\section{Kinematics of the gas}

As proposed by deVaucouleurs and Freeman (1973), the LMC can best be described as a rather flat, disk-shaped system seen nearly face-on. This is confirmed by the shape of the HI $21 \mathrm{~cm}$ line profiles. But, since the spatial distribution of the HI gas in this disk seems to be irregular in a fairly random way - a fact also known from other late type and irregular galaxies - it is doubtful whether the observed gas distribution can be used to derive the inclination $\langle i\rangle$ and orientation $\left\langle\theta_{0}\right\rangle$ of the line-of nodes. Taken from the geometric distribution of other disk tracers (for a summary see Westerlund 1990)

$$
\langle i\rangle=33^{\circ} \pm 6^{\circ},\left\langle\theta_{0}\right\rangle=176^{\circ} \pm 8^{\circ}
$$

where $\langle i\rangle$ is the straight mean of 12 different determinations. However, in recent times, Westerlund prefers $i \approx 45^{\circ}$ as the most likely value.

The young objects, be they stars or gas, all show indications of systematic differential rotation of the disk. If the inner parts of the disk show rigid body rotation, the lines of equal radial velocity should be parallel to the minor axis of the projection, and this can also be used to derive a value for $\theta_{0}$. Values between $181^{\circ}$ (young globular clusters, Freeman et al. 1983) and $221^{\circ}$ (old globular clusters, Freeman et al. 1983) are found, with the HI gas giving values of $208^{\circ}$ or $203^{\circ}$ (Rohlfs $e t$ al. 1984, Rohlfs \& Luks 1990).

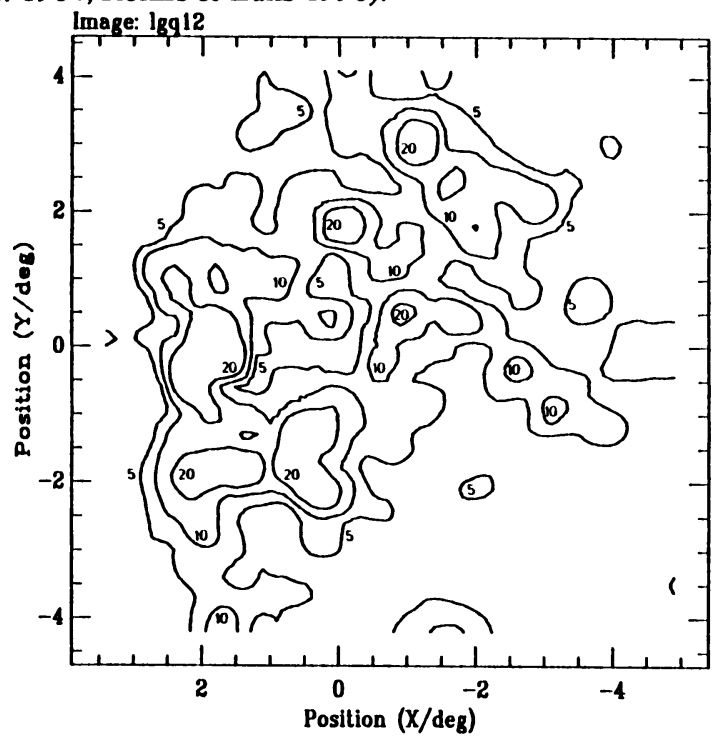

Figure 5. Lines of equal necessity for two Gaussian components in order to represent the measured line profile. 
The difference between the geometric and the kinematic value of $\theta_{0}$ has been explained as being a result of the transverse velocity of the LMC (Feitzinger et al. 1977) where errors of up to $20^{\circ}-30^{\circ}$ are possible. Single peaked HI line profiles indicate gas with a mean weighted radial velocity. But, a fair proportion of the line profiles are double- or even multi-model, indicating that there exist several preferred radial velocities in the column. Defining a measure of how strong the amount of multi-mode-character of a line profile is by dividing the chi-square sum of a twocomponent Gaussian fit to the line profile by that for a single component fit, a comparison of this plot with the total line intensity plot shows that the high-column density parts of the LMC tend to have bi- or multi-modal lineshapes. There is a single component everywhere throughout the LMC region; at the positions of discrete clouds of $\mathrm{HI}$ a second one is added.

An obvious explanation that is supported by a cursory inspection of the radial velocities for these components is that the single pervading component is that of the disk. The additional line components are formed by the gas of separate clouds at different radial velocities. Since no effects of a possible interstellar shock are found, the cloud components, which, after all, have radial velocities differing by up to $40 \mathrm{~km} \mathrm{~s}^{-1}$ from that of the gas of the disk, should be situated above or below the disk, thus indicating a three-dimensional structure. This applies in particular to the large cloud south of 30 Dor. Its radial velocity differs at its eastern edge by $40 \mathrm{~km} \mathrm{~s}^{-1}$ from that of the disk; at its western edge the two velocities agree.

A question not yet resolved concerns the position of 30 Dor in this complex. Is it situated in this cloud or does it belong to the disk proper?

\section{References}

Cohen, R.S., Dame, T.M., Garay, G., Montani, J., Rubio, M., Thaddeus, P. (1988), Astrophys. J. 331, L95.

Davies, R.D., Elliot, K.H., Meaburn, J. (1976), Mem. R. Astron. Soc. 81, 89.

DeVaucouleurs, G., Freeman, K.C. (1973), Vistas in Astron. 14, 163.

Dopita, M.A., Mathewson, D.S., Ford, V.L. (1985), Astrophys. J. 297, 599.

Feitzinger, J.V., Isserstedt, J., Schmidt-Kaler, Th. (1977), Astron. Astrophys. 57, 265.

Freeman, K.C., Illingworth, G., Oemler jr., A. (1983), Astrophys. J. 272, 488.

Martin, N., Prévot, L., Rousseau, J. (1989), Proc. Europ. Colloq. Recent Developments of Magellanic Cloud Research, K.S. de Boer et al. (eds.) (Observatoire de Paris: Paris), p.155.

McGee, R.X., Milton, J.A. (1966a), Aust.J. Phys. 19, 343.

McGee, R.X., Milton, J.A. (1966b), Aust. J. Phys. Astrophys. Suppl. No. 2.

Meaburn, J., Marston, A.P., McGee, R.X., Newton, L.M. (1987), Mon. Not. R. Astron. Soc. 225, 591.

Rohlfs, K., Kreitschmann, J., Siegman, B.C., Feitzinger, J.V. (1984), Astron. Astrophys. 137, 343.

Rohlfs, K., Luks, Th. (1990), in preparation.

Westerlund, B.E. (1964), Observatory 84, 253.

Westerlund, B.E. (1990), Astron. Astrophys. Rev. 2, 29. 